\title{
LOW COST EDUCATIONAL TECHNOLOGY BASED ON OPEN SYSTEM REFERENCE ARCHITECTURE FOR ENGINEERING COURSES
}

\author{
Miguel de Jesús Ramírez Cadena \\ CSIM-ITESM \\ Ave. Eugenio Garza Sada 2501 Sur \\ Monterrey, N.L. 64849 Mexico \\ mdramire@campus.mty.itesm.mx
}

\begin{abstract}
This paper describes a software-based control reference architecture based on the open systems concept use it as a development tool to building common system by a group of users working individually on separated parts of the system. An application of the architecture in engineering courses is showed. In this case, the author had developed an educational frame in computer science, electronic, electrical, mechanic and others related engineering courses where the professor can organize the course in sections in order to be developed by each student or team of students. The concept can be brought to collaborative environments to support virtual organizations where need it multidisciplinary teams.
\end{abstract}

\section{INTRODUCTION}

Open Systems is a concept used to development control systems. The IEEE define open system as follow: "An open system provides capabilities that enable properly implemented applications to run on a variety of platforms from multiple vendors, interoperate with other systems applications and present a consistent style of interaction with the user." (IEEE 1003.0).

The main goals for open system architecture are interoperability, portability, scalability and inter-changeability. Interoperability will only be guaranteed by using standardized data semantics and behavioral models, communication and interaction mechanisms. Portability allows operate the system components on different platforms without any changes. Scalability is a feature to enables the customer to increase or decrease the functionality of a system by upgrading or downgrading specific components. Inter-changeability allows the interchanging of one component 
per another due to its specific capabilities, reliability or performance. Also open system architecture concept is related to internal openness and external openness. The first one refers to openness concerning the internal control functions and the second cover all external interfaces. (Ramírez et al., 2001).

A lot of efforts had been realized in order to create controls based on open systems (Pritschow et al., 2001) :

- Japan. OSEC (Open System Environment for Manufacturing).

- USA. OMAC (Open Modular Architecture Controllers).

- Europe. OSACA (Open System Architecture for Controls within Automation Systems).

- USA-NIST. EMC (Enhanced Machine Controller).

The Manufacturing Integrated Systems Center (CSIM) of the Monterrey Institute of Technology (ITESM) had developed the concept of Universal Numerical Control (CNU) based on open system concept. The rationale of CNU project is related with the fact that in countries like Mexico, small and medium metal-working industry rarely have access to computer numerical control (CNC) machine tools and then they work with conventional machine tools. On the other hand, only well established companies have access to automated machine tools due to its cost. Also the research group has pointed out that Mexican small and medium metal-working industry has been facing major problems in international markets due to lack CNC technology which is the basis for competition in this sector (Phillips et al., 1997).

Therefore an area of opportunity has been identified to develop national technology in the area of CNC using low cost PCs, object oriented programming technologies and software based open system architecture implemented with realtime operating systems. The research group had developed a control prototype used to retrofitted an outdated machine-tool. Actually, five small metalworking enterprises of Mexico had expressed their wish to implement the CNU project on their outdated machines.

The CNU project had created reference architecture based on open systems theory. A reference architecture is a conceptual model that establish rules and methods of integration and standard interfaces between its components in order to reach a structure to let developers build systems with the characteristics established by the architecture (Ramírez (5), 2001).

With the reference architecture is possible design and implement systems formed with different combinations of software modules that can plug and play on the architecture to reach the needed functionality.

Because of the success of the CNU project, an opportunity had been viewed by the author with regards to the open system reference architecture on the scope of real-time systems. The reference architecture really can provide an educational frame in computer science, electronic, electrical, mechanic and others related engineering courses. The professor can organize the course in sections or software 
modules in order to be developed by each student or team of students. Each team needs to follow the specifications of the reference architecture so get communication with the others parts. Each team can do their particular work with independence of the other teams because the reference architecture establishes the communications rules between all parts of the system. Previously, the professor need design the communication rules related with the control system to implement.

The development of low cost educational technology for engineering courses let meeting students with many technical profiles.

\section{EDUCATIONAL REFERENCE ARCHITECTURE}

The proposal to use open system reference architecture to organize engineering courses let the follow:

- Planning build a system by a group of users working individually on separated pieces.

- Using ready-to-use libraries of software in order to development systems.

- Work on the scope of real-time operating systems.

- Prepare the course by the teacher focus on the functionality of each part and the integration of the system.

The real-time item is a key concept of the architecture and let to system respond to internal or external events with an appropriated answer time. The main differences between real-time and standard programming are (Everett, 1995):

- The real-time systems are compacts but complex.

- The real-time make sure the good performance of the systems.

- The real-time programming drive the interrupts scheduler of the systems resources.

The figure 1 show the concept reached by the architecture in order to be use on engineering courses with different profiles of students, this concept get a library of software modules developed by the students which can be integrated to common reference architecture designed by the professor.

This concept of reference architecture is like a "Black Box", where the professor designs the "Black Box" and the students full the box with the necessary modules to reach the functionality designed by the professor. Before starting the course, the professor must to design the interfaces between the different software modules establishing the communications among themselves. 


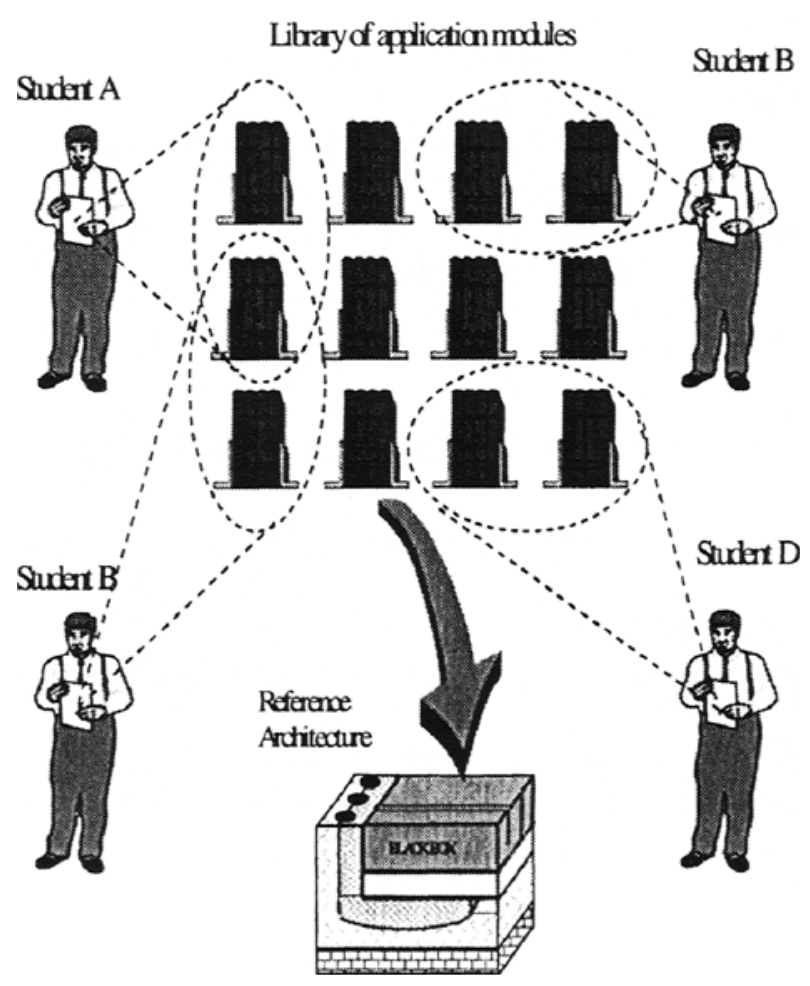

Figure 1. The Reference Architecture Application Concept

The reference architecture offers system software layers to serve as link between applications software modules and hardware. It offer an exactly specified application interface which will provide standard services in the fields of communication, data storage, graphics, dialogue management, configuration and operating system (Ramírez(4), 2001).

The system software layer hide the specific characteristics of processes, operating systems or communication media which are used within the architecture, so that the student always finds the same application interface and just will dedicate effort to develop his part.

\section{APPLICATION ON ENGINEERING COURSES}

The next section shows the organization of electronic engineering course gave in Monterrey Institute of Technology last year. The objective was development a Computer Numerical Control for retrofit a conventional machine-tool (lathe) using 
the reference architecture. Previously, the professor established the application software modules and designed the communications in the architecture. The professor established the architecture with five software modules (see figure 2): External communications module, movement control module, axis control module, auxiliary process module and sensor-actuator module.

The professor must to program three modules belong to the reference architecture: Man-machine module, Configuration system module and Database management module. These modules have the information to manage the application software modules mentioned before.

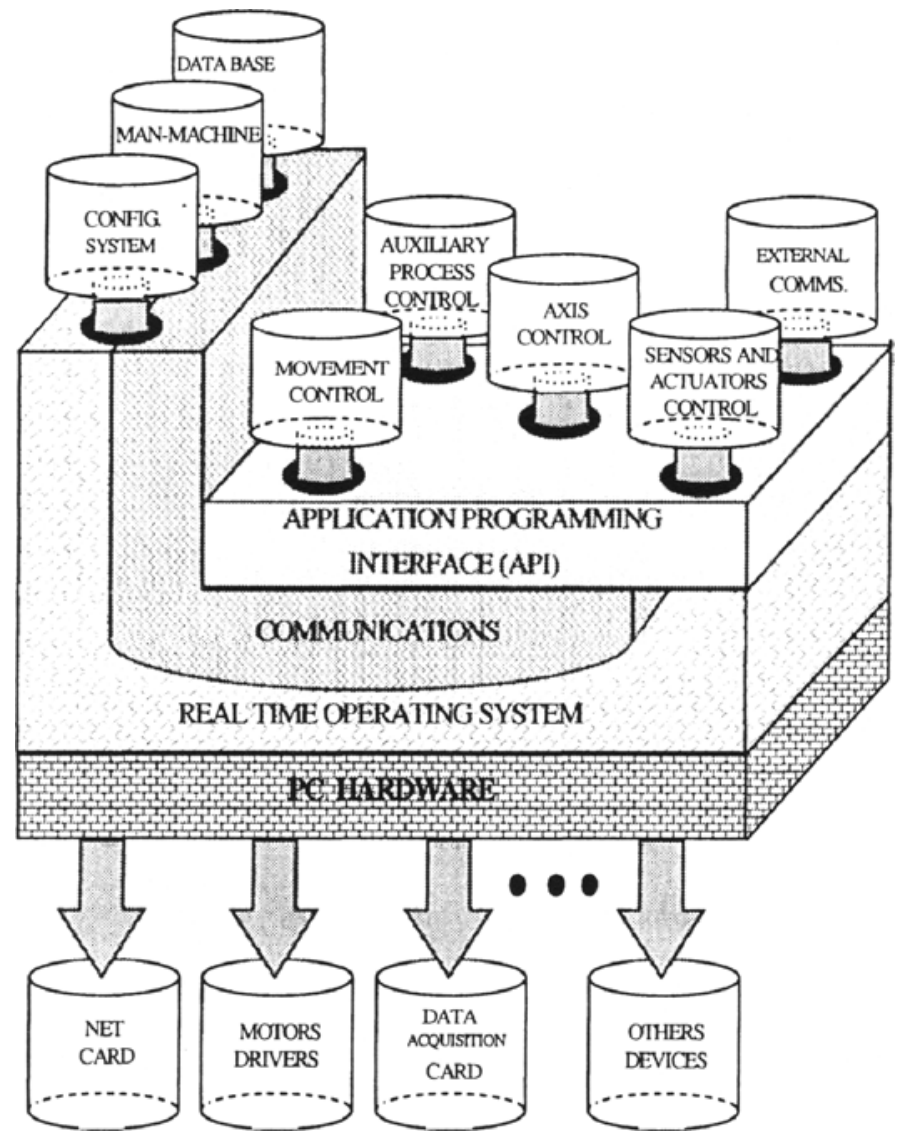

Figure 2. The Reference Architecture of CNU

Ten students of electronic and computer science careers developed the software modules, two students developed each module. In addition, participated three mechanical career students so that help in machining process concepts, mounting equipment on the lathe and machining test pieces.

The information flow for a CNC program is show in figure 3. The flow begins when the user introduce a CNC program in the man-machine interface. The CNC 
code is driven by movement control module to get the basic length unit table. The axis control module processes the basic length unit table to get the signal table for each axis. The auxiliary process module drive the information related with $\mathbf{M}$ codes. The sensors and actuators module converts this information in electrical signals send to machine motors and receive the retrofeed signal of the sensors in the machinetool.

The professor developed a object oriented model using FUSION method and gave, to each development group of module, the document with specific messages that receive and send from/to others modules of the architecture. All modules were programming in $\mathrm{C}++$ using parametric classes (Ramírez et al., 2001).

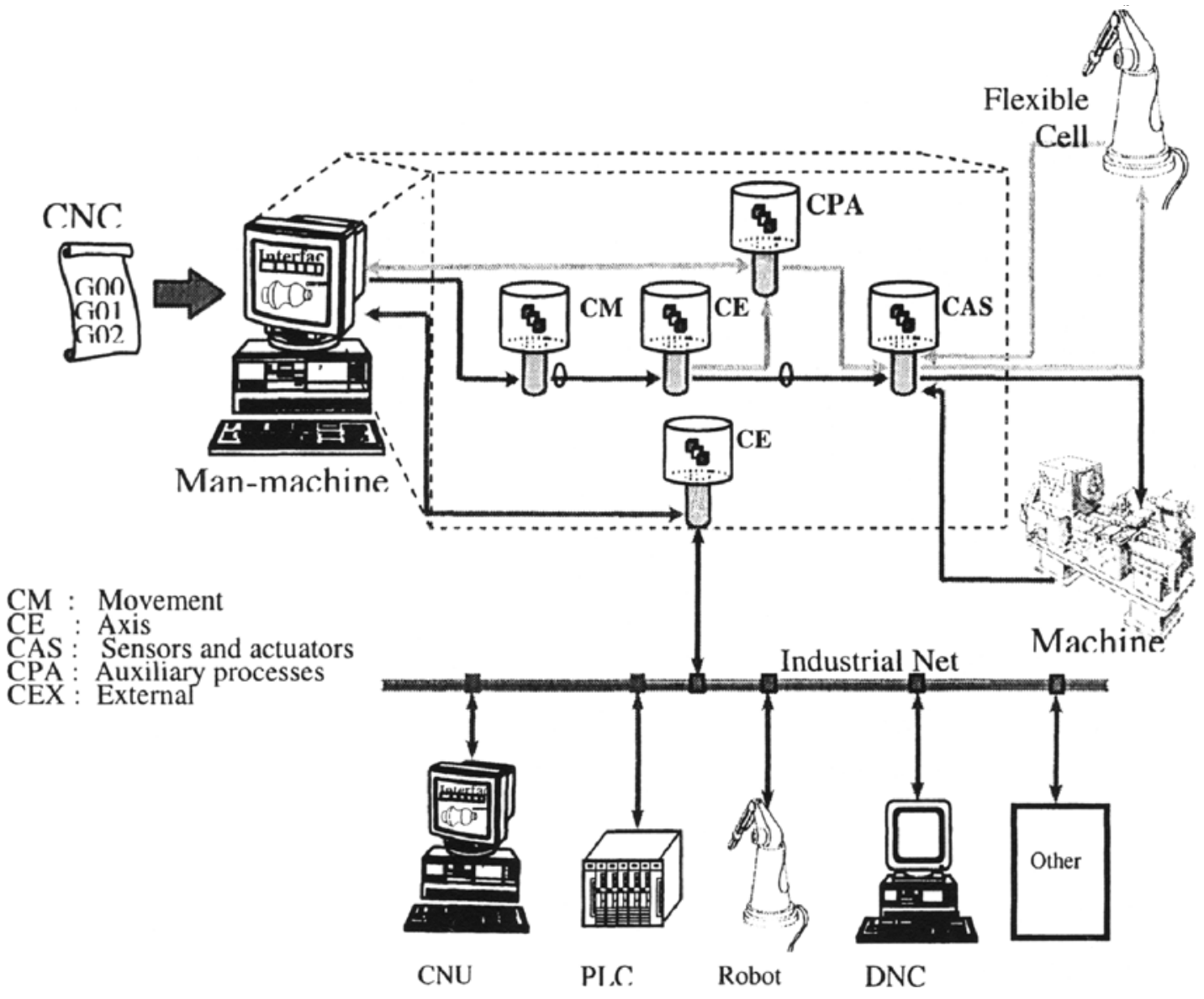

Figure 3. Information Flow in CNU Application.

The lathe was retrofitting mounting servomotor, limit switches, position sensors, a personal computer, ballscrews and data acquisition card. The CNC control implemented used unix platform because all software was developed with QNX real-time operating system. The use of real-time techniques let implemented the system in a standard PC with processor of $600 \mathrm{Mhz}$. 


\section{CONCLUSIONS}

This paper focus on shows the use of reference architecture concept implemented with real-time operating system in order to design and implement any system to do by multidisciplinary teams.

The reference architecture let design and implement an engineering course in order to prove it as an educational tool. The main issues are:

a) The use of the reference architecture let the professor organized the course with flexibility and efficiency.

b) The student centered all his attention in a specific problem or area because the communication forms with others parts of the systems were established previously.

c) This educational tool had proved that is a efficient form to plan engineering courses where participate many persons with different professional profiles.

d) The cost of the implementation of control systems is less than implement the course with commercial platforms of software and expensive machines.

As a future line, the author proposes design and implement a system on the reference architecture relate with virtual organizations to build collaborative environments. Exist on the literature efforts using reference architectures on virtual organizations (Bernus et al., 2002), however, will be interesting to apply real-time items in order to get more control on the system behavior.

\section{ACKNOWLEDGMENTS}

The project has been accepted by the QNX Educational Programm of QNX Software Systems LTD, and therefore the QNX operating system has been given as a grant to our research group. The grant consist development software for the CNU project, the donation consist in the follow items: microkernel of the system, Watcom $\mathrm{C}++$ compilator, Photom microGUI development kit; with a value of 4,200 USD.

\section{REFERENCES}

1. Bernus P., Baltrusch R., Vesterager J., Tolle M. "Fast tracking ICT infrastructure requirements and design, based on enterprise reference architecture and matching reference models". 1st. Edition, Colaborative business ecosystems and virtual enterprises, Kluwer Academic Publishers, 2002. p.p. 293-302.

2. Everett W.W. "Reliability and safety of real-time systems", Computer. May 1995. p.p. 13-16.

3. Phillips L., Valero J., Tijerina J. "Bienes de producción críticos en la industria manufacturera del estado de Nuevo León: el caso de los tornos de control numérico”. Entorno económico Journal. Vol. XXXV. June 1997. p.p. 13-16.

4. Pritschow G., Altintas Y., Jovane F., Koren Y., Mitsuishi M., Takata S., Van Brussel H., Weck M., Yamazaki K. “Open Controller Architecture: Past, Present and Future. CIRP. 2001. 
5. Ramírez M., "Algoritmos de interpolación matemáticos aplicados al corte de metal". Memories of the First meeting in Mathematics applied to the Engineer, computing and science, San José, Costa Rica. February 6-9, 2001.

6. Ramírez M., "Desarrollo de Geotecnología: El caso del control Numérico Universal”. Memories of the IX seminario Latino-Americano de Gestión Tecnológica ALTEC 2001, San José, Costa Rica. October 17-19, 2001.

7. Ramírez M., Molina A., "Sofware based Computer Numerical Controller for Low Cost Automation in Small and Medium sized Metal-Processing Enterprises in Developing Countries". Memories of the VI IFAC Symposium on Cost Oriented Automation, Berlin, German. October 8-9, 2001.

8. IEEE 1003.0, Technical Committee of Open Systems of IEEE. 